\title{
Coexistence of Multiple Nonlinear States in a Tristable Passive Kerr Resonator
}

\author{
Miles Anderson, ${ }^{*}$ Yadong Wang, François Leo, ${ }^{\dagger}$ Stéphane Coen, Miro Erkintalo, ${ }^{\ddagger}$ and Stuart G. Murdoch ${ }^{\S}$ \\ The Dodd-Walls Centre for Photonic and Quantum Technologies, Department of Physics, \\ The University of Auckland, Auckland 1142, New Zealand \\ (Received 1 February 2017; revised manuscript received 18 May 2017; published 15 August 2017)
}

\begin{abstract}
Passive Kerr cavities driven by coherent laser fields display a rich landscape of nonlinear physics, including bistability, pattern formation, and localized dissipative structures (solitons). Their conceptual simplicity has for several decades offered an unprecedented window into nonlinear cavity dynamics, providing insights into numerous systems and applications ranging from all-optical memory devices to microresonator frequency combs. Yet despite the decades of study, a recent theoretical work has surprisingly alluded to an entirely new and unexplored paradigm in the regime where nonlinearly tilted cavity resonances overlap with one another [T. Hansson and S. Wabnitz, J. Opt. Soc. Am. B 32, 1259 (2015)]. We use synchronously driven fiber ring resonators to experimentally access this regime and observe the rise of new nonlinear dissipative states. Specifically, we observe, for the first time to the best of our knowledge, the stable coexistence of temporal Kerr cavity solitons and extended modulation instability (Turing) patterns, and perform real-time measurements that unveil the dynamics of the ensuing nonlinear structure. When operating in the regime of continuous wave tristability, we further observe the coexistence of two distinct cavity soliton states, one of which can be identified as a "super" cavity soliton, as predicted by Hansson and Wabnitz. Our experimental findings are in excellent agreement with theoretical analyses and numerical simulations of the infinite-dimensional Ikeda map that governs the cavity dynamics. The results from our work reveal that experimental systems can support complex combinations of distinct nonlinear states, and they could have practical implications to future microresonator-based frequency comb sources.
\end{abstract}

DOI: 10.1103/PhysRevX.7.031031

Subject Areas: Nonlinear Dynamics,Optics,Photonics

\section{INTRODUCTION}

Beginning with theoretical studies of bistability [1], the behavior and dynamics of externally driven nonlinear optical cavities have been extensively investigated for almost 50 years. Besides many application prospectsranging from all-optical information storage [2-5] to photonic computing [6,7]— the continuous interest in such systems stems from the diversity of universal nonlinear physics they support [8]. Pattern formation and selforganization [9-13], dissipative solitons [14-20], chaos [21-24], vortices [25-27], and topological phase solitons [28] all represent examples of the richness of nonlinear cavity physics.

\footnotetext{
*Present address: École Polytechnique Fédérale de Lausanne (EPFL), CH-1015 Lausanne, Switzerland.

${ }^{\dagger}$ Present address: Université libre de Bruxelles, 50 Avenue F.D. Roosevelt, CP 194/5, B-1050 Bruxelles, Belgium.

*m.erkintalo@auckland.ac.nz

§s.murdoch@auckland.ac.nz

Published by the American Physical Society under the terms of the Creative Commons Attribution 4.0 International license. Further distribution of this work must maintain attribution to the author(s) and the published article's title, journal citation, and DOI.
}

The simplest nonlinear optical cavity, which nevertheless captures much of the principal dynamics, is arguably that of the passive Kerr cavity. For several decades, the Kerr cavity model has offered an unparalleled window into complex cavity dynamics. It has played a particularly "decisive role in promoting the field of optical pattern formation" [8], and in elucidating the intimately related emergence of localized dissipative structures commonly referred to (in optics) as cavity solitons (CSs) [14,15]. Such CSs correspond to localized wave packets that sit on top of a nonzero homogeneous background, and they have been subject to significant research efforts due to their application prospects as bits in all-optical buffers and processing units (for comprehensive reviews, see Refs. [14-16,29]). Studies focused initially on spatial CSs [2], which can manifest themselves as persisting spots in diffractive nonlinear systems, such as semiconductor microcavities [4]. More recently, however, the spotlight has shifted to dispersive systems and temporal CSs [3,29]: pulses of light circulating in optical ring resonators. While first observed [30] and studied [31-33] in macroscopic resonators constructed from single-mode optical fibers, the interest in temporal CSs has surged over the past couple of years with the identification of their key role in the generation of stable frequency combs in optical microresonators [34-40]. Such frequency combs have several potential applications 
in, e.g., telecommunications and spectroscopy [41-45], fueling continuous efforts to better understand the dynamics that underpin CSs in dispersive Kerr resonators [46-57].

CSs are typically explained to arise under conditions of coexistence between a periodic pattern and a stable homogeneous state $[29,58]$; they correspond to localized excitations that connect one cycle of the pattern with the homogeneous state [59]. In a pure Kerr cavity, suitable conditions can be readily met in the region of continuous wave (cw) bistability [60], which arises from the tilt of the Lorentzian cavity resonances induced by the Kerrnonlinear phase shift. But of course, the fact that cavity resonances repeat periodically elicits the following question: What if the cavity driving is so strong that the nonlinear phase shift exceeds $2 \pi$, i.e., such that adjacent spectral resonances actually overlap [as in Fig. 1(a)]? Despite the decades of study into passive Kerr resonators and CSs, this question has remained virtually unstudied. It is only recently that Hansson and Wabnitz theoretically considered some of the implications of such strong cavity driving [61], motivated by the large phase shifts (of the order $\pi$ ) already demonstrated in microresonator frequency comb experiments [62]. Significantly, the authors predicted that, under conditions of $\mathrm{cw}$ tristability, two different CS states may simultaneously coexist. Because one of the CS states was predicted to possess a significantly shorter duration than the other-and thus be more attractive for broadband frequency comb generation-they coined the term "super" CS for its description [61].

In this article, we report on the first combined experimental and theoretical study of passive Kerr cavity dynamics in the strong-driving regime, as characterized by nonlinear phase shifts in the vicinity of $2 \pi$ and beyond. Our experiments are performed using synchronously driven optical fiber ring resonators, and we observe new nonlinear behaviors emblematic of the strong-driving regime: coexistence of distinct dissipative structures associated with adjacent, nonlinearly overlapping cavity resonances. In particular, we predict and observe-both for the first time to our knowledge-localized Kerr CSs sitting atop periodic Turing patterns arising from the modulation instability of the underlying homogeneous state. Furthermore, we report the first experimental observations of the coexistence of two distinct CS states, as predicted earlier by Hansson and Wabnitz [61]. All of our experimental results are in excellent agreement with numerical simulations of the infinite-dimensional Ikeda map that governs the cavity dynamics, and they conform to a simple physical interpretation of coexistence of nonlinear states associated with individual resonances. Notably, although the full "mixed" states we observe are beyond the standard mean-field analysis of passive Kerr cavities [10,63], we find remarkably that their constituent nonlinear structures are not.
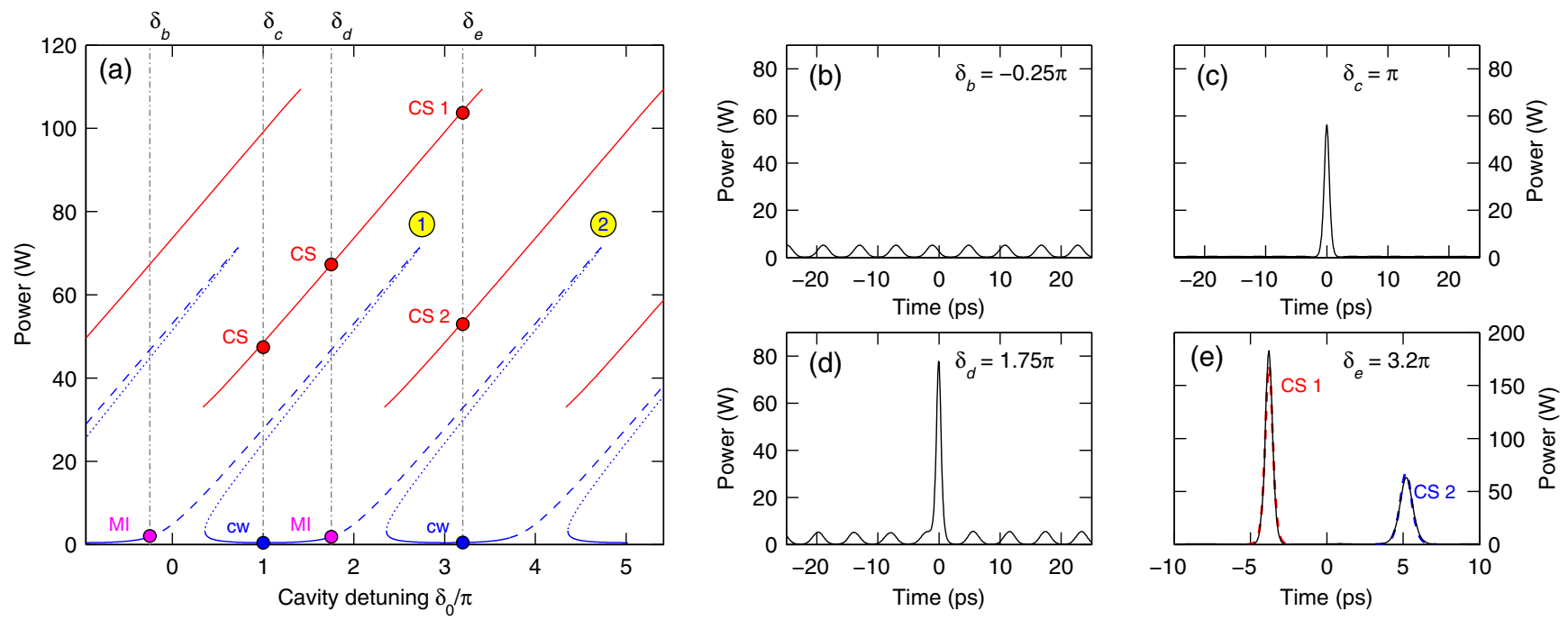

FIG. 1. Cavity resonances and examples of nonlinear structures. (a) Blue curves at the bottom show power levels of cw steady-state solutions, while red curves at the top show CS branches predicted individually for the different resonances by the mean-field LugiatoLefever equation (LLE) (see Appendix B). For clarity, the CS branches are plotted as $f\left(\delta_{0}\right)=10 \mathrm{~W}+P_{p}\left(\delta_{0}\right) / 2$, where $P_{p}\left(\delta_{0}\right)$ is the soliton peak power. (b)-(e) Examples of steady-state nonlinear structures obtained from numerical simulations of the Ikeda map at different detunings [highlighted in (a) as dash-dotted vertical lines]: (b) MI pattern, $\delta_{0}=1.75 \pi-2 \pi=-0.25 \pi$, (c) CS on a cw background, $\delta_{0}=\pi$, (d) CS coexisting with a MI pattern, $\delta_{0}=1.75 \pi$, (e) coexistence of two CSs associated with adjacent resonances, $\delta_{0}=3.2 \pi$. In (e), the dashed red and blue curves correspond to CS profiles predicted individually for the different resonances by the LLE. The parameters used in all of the calculations are $\theta=0.1, P_{\text {in }}=15 \mathrm{~W}, \rho=0.73, \beta_{2}=-22 \mathrm{ps}^{2} / \mathrm{km} \gamma=1.2 \mathrm{~W}-1 \mathrm{~km}^{-1}$, $L=100 \mathrm{~m}$. The mean-field results use $\alpha=0.145$, corresponding approximately to half the total cavity losses per round-trip (see Appendix B). Note the different axes in (b)-(d) and (e), highlighting the much larger power and shorter duration of the "super" CS. 


\section{THEORY}

The experiments that follow are based on an optical fiber ring resonator that is coherently driven by quasi-cw laser light. Several prior studies, performed in the regime of comparatively small nonlinear phase shifts, have demonstrated such systems to be ideal test beds for the experimental exploration of passive Kerr cavity phenomena $[30,31,33,54,64,65]$. We begin by briefly recalling the model equations that govern the system behavior as well as the steady-state solutions they are known to support. We then argue and demonstrate by means of numerical simulations how new combinations of nonlinear states can emerge when adjacent resonances overlap.

\section{A. Model equations}

The evolution of the slowly varying electric field envelope in a coherently driven fiber ring resonator is governed by a (generalized) Ikeda map [35,61,63,66,67]. In the beginning of each round-trip, a cw driving field $E_{\text {in }}$ with power $P_{\text {in }}=\left|E_{\text {in }}\right|^{2}$ is coherently superimposed on the lightwave circulating in the resonator, such that the timedomain electric field envelope $E_{m+1}(z, \tau)$ at the beginning of the $(m+1)$ th cavity transit obeys the following boundary condition:

$$
E_{m+1}(z=0, \tau)=\sqrt{\theta} E_{\mathrm{in}}+\sqrt{\rho} E_{m}(z=L, \tau) e^{-i \delta_{0}} .
$$

Here, $z$ is the longitudinal coordinate along the optical fiber forming the resonator, $\tau$ is time defined in a reference frame moving at the group velocity of light in the fiber, $\theta$ is the power transmission coefficient of the coupler (located at $z=0$ ) used to inject the driving field $E_{\text {in }}$ into the cavity, $L$ is the round-trip length of the resonator, and $\delta_{0}$ is the phase detuning between the driving field and a cavity resonance. For simplicity, we lump all dissipation (arising, e.g., from the input coupler, fiber absorption, or component loss along the fiber loop) in the boundary condition, with $1-\rho$ describing the total power lost per round-trip; the parameter $\rho$ is fully determined by the cavity finesse $\mathcal{F}$, with $\rho \approx 1-2 \pi / \mathcal{F}$ (valid in the limit $\mathcal{F} \gg 1$ ). With this approximation, the field envelope $E_{m}(z=L, \tau)$ at the end of the $m$ th cavity transit can be obtained by numerically integrating a generalized nonlinear Schrödinger equation [68],

$$
\frac{\partial E_{m}(z, \tau)}{\partial z}=-i \frac{\beta_{2}}{2} \frac{\partial^{2} E_{m}}{\partial \tau^{2}}+i \gamma\left[R(\tau) *\left|E_{m}\right|^{2}\right] E_{m},
$$

where $\beta_{2}$ is the group-velocity dispersion (GVD) coefficient, $\gamma$ is the nonlinearity coefficient, and $R(\tau)=$ $\left(1-f_{R}\right) \delta(\tau)+f_{R} h_{R}(\tau)$ is the nonlinear response function that includes both the instantaneous Kerr nonlinearity $[\delta(\tau)$ is the Dirac delta function] and stimulated Raman scattering (SRS), with $f_{R}$ the Raman fraction of the nonlinearity (for silica glass, $f_{R}=0.18$ ) and $h_{R}(\tau)$ the Raman response function [69].

As we see below, SRS is key to fully explaining our experimental observations; however, its role is to merely perturb the nonlinear states supported by pure Kerr cavity dynamics (see Appendix A). Accordingly, we begin our discussion by neglecting SRS and set $f_{R}=0$. In this limit, Eqs. (1) and (2) describe a dispersive cavity with a purely instantaneous Kerr nonlinearity. Because of the equivalence between paraxial-beam diffraction and dispersive pulse spreading [70], the system is analogous to a spatially diffractive Kerr cavity, and can thus be considered a generic representation of a one-dimensional Kerr cavity. We remark in this context that, under specific conditions, Eqs. (1) and (2) can be averaged (see Appendix B) into a single meanfield equation [63] that is fully analogous to the celebrated Lugiato-Lefever equation (LLE) of spatially diffractive cavities [10]. Although our experimental conditions are beyond such a mean-field approximation, the LLE nevertheless provides important insights (as we discuss below).

\section{B. Coexistence of multiple nonlinear states}

We are interested in the regime of anomalous dispersion $\left(\beta_{2}<0\right)$, where the nonlinear Schrödinger equation Eq. (2) is self-focusing (for silica fibers, $\gamma>0$ ). In this regime, passive Kerr cavities are well known to support three families of nonlinear states, each of which correspond to a distinct steady-state solution of the Ikeda map [60]. These are the (i) homogeneous cw states, (ii) periodic (Turing) patterns, and (iii) localized CSs. The different states are closely interrelated: patterned states arise from the modulation instability (MI) of a cw state, while CSs can be understood as combinations of patterned and cw states, corresponding to singular cycles of the pattern sitting atop a cw background [29]. To illustrate how new combinations can emerge when the driving is so strong that adjacent resonances overlap, we plot in Fig. 1(a) the cw steady-state solutions of the Ikeda map (blue curves; see also Appendix C) for parameters similar to the experiments that follow (listed in the caption of Fig. 1). Here, dotted lines correspond to states that are unconditionally unstable (and are not considered further), while dashed lines highlight states that exhibit MI. Also shown (as red curves) are the CS branches predicted for each individual resonance based on the mean-field LLE [30,60]. For clarity, only the upper branch of the CS solution is shown; the lower branch, which bifurcates subcritically from the lower $\mathrm{cw}$ state, is unconditionally unstable and will not be discussed further. In the upper branch, the CSs exhibit self-pulsing instabilities (associated with a Hopf bifurcation) for small (relative) detunings [15,31,54]. As these instabilities do not influence our main findings, they are not discussed further.

The $\mathrm{cw}$ solutions in Fig. 1(a) represent periodically repeating cavity resonances that are tilted due to the Kerr 
nonlinearity (for the parameters we use, the Kerr tilt is $\left.\phi_{\mathrm{NL}} \approx 2.7 \pi\right)$. Besides those solutions, inspection of Fig. 1(a) allows us to identify four possible combinations of nonlinear states, and in Figs. 1(b)-1(e), we show results from direct numerical simulations of the Ikeda map that illustrate these different behaviors [71]. The simulations all use different cavity detunings [labeled $\delta_{\text {b-e }}$ in Fig. 1(a)] and initial conditions (MI states grow from random noise while CSs are excited by assuming an initial condition corresponding to a suitable sech profile on a $\mathrm{cw}$ background).

First, referring to resonance (1), as labeled in Fig. 1(a), MI analysis of the Ikeda map [67] reveals that the upper cw state exhibits MI for cavity detunings $\delta_{1}=\delta_{0} \gtrsim \delta_{\mathrm{MI}}=-0.30 \pi$. Indeed, at the cavity detuning $\delta_{\mathrm{b}}=-0.25 \pi$, periodic patterns emerge from an initially noisy background [Fig. 1(b)]. When the detuning increases beyond the up-switching point, which marks the lower boundary of cw bistability, CSs can be expected $[30,60]$. For moderate detunings $\left(\delta_{0}<2 \pi\right)$, they correspond to the standard CSs that sit on top of a cw background [Fig. 1(c), $\delta_{\mathrm{c}}=\pi$ ], with the background coinciding precisely with the stable lower cw state of the resonance (being the only stable $\mathrm{cw}$ state available). However, in the strong driving regime, where adjacent resonances overlap, the lower state of the first resonance eventually morphs into the upper state of its neighbouring resonance [labeled (2) in Fig. 1(a)], and can therefore be expected to exhibit MI. Remarkably, as evidenced by the Ikeda-map simulation for $\delta_{\mathrm{d}}=1.75 \pi$, CSs continue to exist in this region [Fig. 1(d)]. They now sit, however, on top of a background that is not $\mathrm{cw}$, but rather a periodic Turing pattern ensuing from the MI of the upper branch of the second resonance (being the only background state available).

The possibility of CSs existing atop modulated backgrounds has been previously noted in other contexts [72-75]. In particular, Hachair et al. have shown experimental and theoretical evidence of spatial CSs sitting atop a spatially homogeneous background that exhibits slow temporal oscillations due to an underlying Hopf instability [72], while Hansson and Wabnitz have theoretically identified temporal CSs in a dual-pumped system where the modulated background is simply due to the beating of two separate driving fields [73]. Both of these scenarios are fundamentally different from that shown in Fig. 1(d), where the rapid modulation ensues from the Turing (or modulation) instability of the homogeneous state, and thus corresponds to an extended (nonhomogeneous) nonlinear dissipative structure in its own right. Interestingly, we find that the CSs do not, in general, sit at the extrema of the pattern. For example, for the parameters used in Fig. 1(d), the soliton is found slightly offset from a maximum. Full analysis of this behavior and its parameter dependence is beyond the scope of our current work, but we remark that the resulting asymmetric field profiles bear resemblance to observations of spontaneous symmetry breaking in passive Kerr cavities driven by short pulses [76].

Finally, if the driving is sufficiently strong, such that the CS branch from the first resonance extends into the region of cw bistability of the second resonance, a situation may arise where the CS solutions associated with two adjacent resonances can coexist, as predicted in Ref. [61]. In this regime, the intracavity field is composed of two different CS states with distinct characteristics (duration, peak power), both of which sit on top of the lower state $\mathrm{cw}$ solution of the second resonance [Fig. 1(e), $\delta_{\mathrm{e}}=3.2 \pi$ ].

The standard mean-field analysis of passive Kerr cavitiesbased on the simple LLE $[10,63]$ with fully distributed driving and loss [see Appendix B] - is unable to capture the full mixed nonlinear states associated with overlapping resonances [Figs. 1(d) and 1(e)]. This can be readily understood by recalling that the cw response of the LLE corresponds to a unique Lorentzian resonance [see Appendixes B and $\mathrm{C}$ ], fundamentally limiting the model's reach to states associated with a single resonance (i.e., MI patterns or CSs atop a cw background). In this context, we reemphasize that the CS branches shown in Fig. 1(a) are obtained individually for each resonance using the LLE, and should therefore be understood as qualitative predictions only. Somewhat surprisingly, however, we find that, although the full mixed states are beyond the simple LLE, the constituent nonlinear states are not, despite the large absolute detunings. For example, the dashed red and blue curves in Fig. 1(e) show CS profiles predicted by the LLE for two different cavity detunings, as measured from the centers of the respective linear resonances $\left(\delta_{1}=\delta_{0}=3.2 \pi\right.$ and $\delta_{2}=\delta_{1}-2 \pi=$ $1.2 \pi)$. As can be seen, the individual profiles predicted by the LLE are in excellent agreement with the CSs that make up the full mixed state obtained from the Ikeda map (black curves). This is an unexpected result: the LLE is derived with the assumption $\delta_{0} \ll 1$, yet here we find it correctly predicting the characteristics of individual CSs even when $\delta_{0}>2 \pi$. Although the LLE cannot capture the coexistence of different nonlinear states, this finding further consolidates its broad usefulness even outside the regions where it can be considered strictly valid. For example, we can now readily explain the different temporal durations of the two CSs shown in Fig. 1(e): the LLE predicts the width of a CS to scale as $\Delta \tau \propto \delta_{0}^{-1 / 2}$ [60], and so the soliton associated with the first resonance expectedly possesses a significantly shorter duration (and broader spectrum) than the one associated with the second resonance. The term "super" CS was coined by the authors of Ref. [61] to highlight this difference, yet we emphasize that the two solitons should be understood as the same structures at different detunings [as apparent from Fig. 1(a)].

\section{EXPERIMENTAL SETUPS}

To experimentally study the existence of the new mixed nonlinear states, a passive Kerr cavity platform capable of 
generating large nonlinear phase shifts of the order of $2 \pi$ is required. As $\phi_{\mathrm{NL}} \approx \theta P_{\text {in }} \gamma L \mathcal{F}^{2} / \pi^{2}$, this calls for a strongly driven cavity with a high finesse and a long roundtrip length. We achieve suitable conditions by using macroscopic fiber ring resonators that are synchronously driven by quasi-cw pulses. Although similar systems have previously been used to successfully study Kerr cavity dynamics $[54,65,67]$, they have not been optimized to allow access to the highly nonlinear regime where adjacent resonances overlap.

Our experiments are performed using two different fiber ring resonators, both of which consist of a loop of optical fiber closed on itself with a fiber coupler. The first resonator is similar to the one used in Refs. [32,33]. It is built around a 90/10 coupler, is $100 \mathrm{~m}$ long, and incorporates an optical isolator to inhibit stimulated Brillouin scattering (SBS) and a wavelength-division multiplexer that does not play a role in the experiments we report here. The cavity has a total measured finesse of 20 ( $\rho \approx 0.73$ ), which corresponds to $27 \%$ losses per round-trip. This cavity is ideal for the study of coexistence between a CS and a MI pattern; however, its limited length and finesse prevent access to the regime where two distinct CS states coexist. Our second cavity has been custom-built to overcome this issue. It uses a 95/5 coupler, has a total length of $300 \mathrm{~m}$, and does not include an isolator or a wavelength-division multiplexer, thereby yielding a higher finesse of about $48(\rho \approx 0.88)$. Both cavities are composed entirely of standard telecommunication singlemode optical fiber (SMF-28) with nonlinearity and groupvelocity dispersion coefficients $\gamma=1.2 \mathrm{~W}^{-1} \mathrm{~km}^{-1}$ and $\beta_{2}=-22 \mathrm{ps}^{2} \mathrm{~km}^{-1}$ (at $1550 \mathrm{~nm}$ ), respectively.

The two different resonators offer complementary advantages for the study of different nonlinear structures. In particular, while the 300-m-long cavity in principle allows access to all the different regimes of interest, we find that its long length and high finesse obstruct the active stabilization of the cavity detuning. As the detailed study of coexisting CS and MI states greatly benefits from such stabilization, the 100 -m-long cavity is more ideal for the exploration of that regime. As we show below, the study of coexisting CS states does not critically require active stabilization, and can thus be satisfactorily accomplished using our 300-m-long cavity.

Each of the cavities is driven by quasi-cw pulses synchronized to their respective round-trip time. The driving pulses are generated by passing the output of a narrow linewidth, $1550 \mathrm{~nm}$, distributed feedback, cw fiber laser through an intensity modulator, followed by a $2-\mathrm{W}$ erbium-doped fiber amplifier [54]. After spectral filtering to remove the amplified spontaneous emission component of the signal, we obtain flattop pulses with 4-10-ns duration and a peak power up to $10 \mathrm{~W}$. Thanks to the large peak power of the quasi-cw driving pulses, very large nonlinear phase shifts can be induced. For the 100-m-long cavity, we estimate the maximum phase shift to be around $2.1 \pi$; for the 300-m-long cavity, we can reach phase shifts in excess of $4 \pi$. At this point, we note that the driving pulses are sufficiently short to mitigate the detrimental effects of SBS, and we do not observe any signatures of SBS in our experiments. In fact, this represents a key feature in our experiments: the high finesse of our 300-m-long cavity is precisely underpinned by the absence of an optical isolator that is required for SBS suppression under pure $\mathrm{cw}$ driving [30].

To directly monitor the Kerr cavity dynamics, both resonators include a 99/1 tap coupler whose $1 \%$ output port permits direct measurement of the intracavity field. This field is characterized using either an optical spectrum analyzer, frequency-resolved optical gating (FROG) or a real-time measurement system consisting of a $12.5-\mathrm{GHz}$ amplified photodiode and a $40 \mathrm{GSa} / \mathrm{s}$ real-time oscilloscope. At about $50 \mathrm{ps}$, the temporal resolution of this realtime measurement system is significantly longer than the picosecond time scales of the CS and MI patterns we wish to observe. Nonetheless, as we see below, provided that individual CSs remain separated from one another by more than this resolution, it is possible to clearly observe the system's dynamical evolution and to infer the emergence of new nonlinear mixed states.

\section{RESULTS}

\section{A. Coexistence of CSs and MI patterns}

We first describe results we obtain using our 100-m-long cavity, namely, the observation of temporal CSs sitting on a periodic MI pattern [as in Fig. 1(d)]. In these experiments, we set the pump power of the nanosecond driving pulses to about $10 \mathrm{~W}$, which induces a nonlinear Kerr tilt of about $2.1 \pi$, thus permitting the overlap of two consecutive resonances. To stabilize the phase detuning $\delta_{0}$, we use a proportional-integral-derivative servo system that monitors the average power exiting the resonator at the 99/1 tap coupler (locking the average output power to a set level locks the detuning [32]).

MI analysis of the Ikeda map [67] predicts that, for our experimental conditions, an individual resonance will support patterned MI states for detunings larger than a threshold value of $\delta_{2} \approx-0.25 \pi$, corresponding to $\delta_{1}=$ $2 \pi+\delta_{2} \approx 1.75 \pi$ relative to the preceding resonance. For the sake of discussion, we start by demonstrating the standard configuration of a CS sitting atop a cw background [as in Fig. 1(c)], and lock the pump detuning just below the MI threshold, at $\delta_{2} \approx-0.29 \pi\left(\delta_{1} \approx 1.71 \pi\right)$. The blue curve in Fig. 2(a) shows the optical spectrum measured at the output of the 99/1 tap coupler before a CS is excited. As can be seen, the spectrum is composed of a single component at the pump wavelength, evidencing a quasi-cw intracavity field. We then excite a temporal CS corresponding to the first resonance (with detuning $\delta_{1}=1.71 \pi$ ) by abruptly perturbing the system. Specifically, by cycling the sign of the proportional component of the 

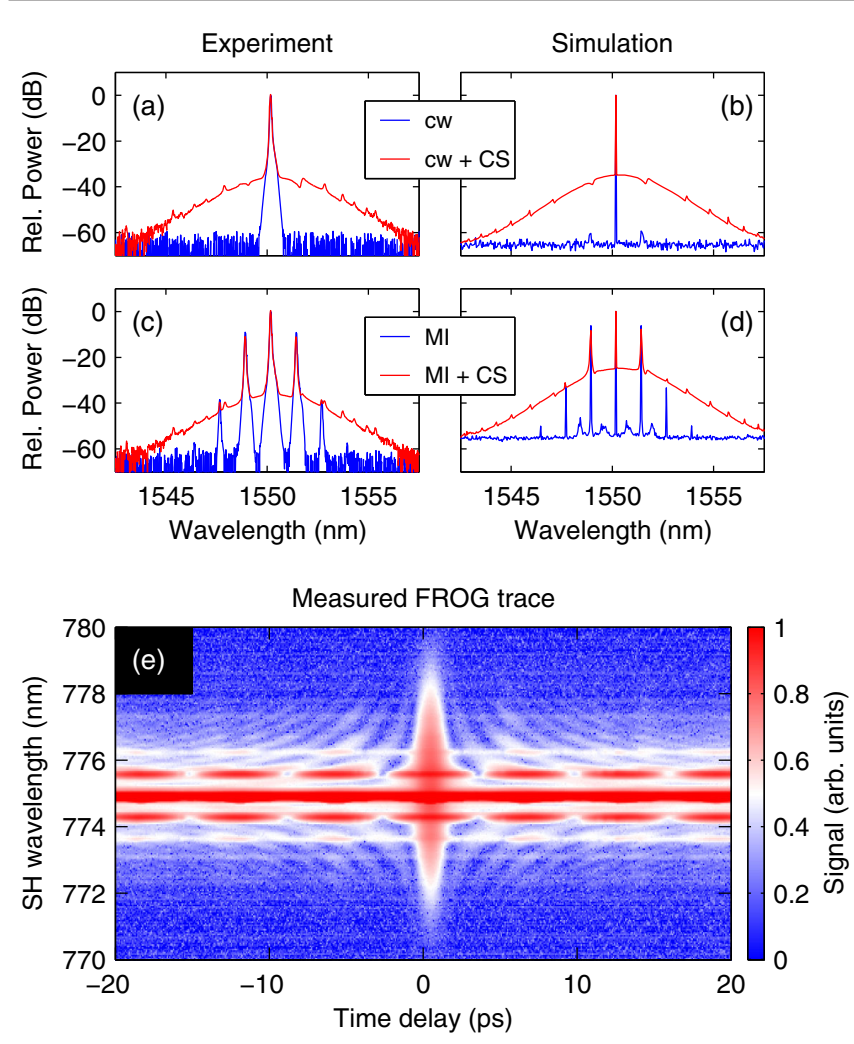

FIG. 2. Experimental evidence of coexisting CSs and MI patterns. (a) Experimentally measured spectra at the cavity output before (blue curve) and after (red curve) the excitation of a CS, with the cavity detuning set below the MI threshold of the second resonance ( $\delta_{1} \approx 1.71 \pi ; \delta_{2} \approx-0.29 \pi$ ). (b) Corresponding numerical simulations. (c) Same as (a) but with the detuning set above the MI threshold of the second resonance $\left(\delta_{1} \approx 1.79 \pi\right.$; $\delta_{2} \approx-0.21 \pi$ ). (d) Numerical simulations corresponding to (c). (e) Experimentally measured FROG trace corresponding to the spectrum shown in (c). SH: second-harmonic.

proportional-integral-derivative servo's output, we rapidly (within $100 \mathrm{~ms}$ ) sweep the cavity detuning towards the zero of the first resonance and then back to its original value. (As in Refs. [34,49], CSs are excited as the detuning is scanned back to the original value.) After this perturbation, the spectrum measured at the cavity output [red curve in Fig. 2(a)] clearly shows a broad $\operatorname{sech}^{2}$-shaped feature indicative of a temporal CS, superimposed on top of the original quasi-cw field. Figure 2(b) shows corresponding spectra obtained from numerical simulations (parameters as quoted above), and we can see very good agreement with experimental observations. [This simulation, and all the simulations that follow, is obtained from Eqs. (1) and (2) using experimental parameters quoted previously, and with SRS included with $f_{R}=0.18$.] Note that the quasi$\mathrm{cW}$ components appear broader in our experimentally measured spectra simply due to the finite $(\sim 50 \mathrm{pm})$ resolution of our optical spectrum analyzer. We also note that most of the small dips and peaks in the CS spectrum (visible in both measurements and simulations) correspond to Kelly-like sidebands arising from the cavity periodicity: their spectral positions agree with wavelengths extracted from well-known phase-matching conditions [47,77]. Polarization-resolved measurements reveal that small additional peaks, present only in the experimentally measured spectra, arise from the coupling between two orthogonal polarization states, as recently detailed in Ref. [78]. Corroborating evidence is presented in Ref. [79].

To now demonstrate the new combination of a CS and a MI pattern [as predicted in Fig. 1(d)], we repeat the experiment above but with the cavity detuning locked just above the threshold of MI of the second resonance at $\delta_{2} \approx-0.21 \pi\left(\delta_{1} \approx 1.79 \pi\right)$. Similar to Fig. 2(a), the blue curve in Fig. 2(c) shows the measured spectrum in the absence of CSs. In agreement with the prediction that no stable cw state should exist, we see clear spectral signatures of a MI pattern: sidebands equally spaced by $160 \mathrm{GHz}$. We then excite a CS using the same detuning-sweep approach as above, and the red curve in Fig. 2(c) shows the spectrum measured after the perturbation. Remarkably, we again see the broad sech ${ }^{2}$-shaped feature characteristic of a CS, but now superimposed on top of the original MI pattern. Figure 2(d) shows the spectrum of a numerically simulated state corresponding to a CS atop a MI pattern, and we see very good agreement with experimental observations. In addition to spectral measurements, we also record the FROG trace of this state. This is shown in Fig. 2(e), and consists of two components: a temporally extended, modulated structure with a period of $6.3 \mathrm{ps}$, and an isolated picosecond pulse centered around the zero delay. The trace is clearly consistent with an intracavity field akin to that shown in Fig. 1(d), i.e., a picosecond-scale temporal CS surrounded by a $160-\mathrm{GHz}$ MI pattern. Our experiments show that this state persists as long as the cavity detuning stays locked, which typically corresponds to a time scale of several minutes (equivalent to hundreds of millions of photon lifetimes).

To further confirm that the results in Figs. 2(c) and 2(e) correspond to a genuine mixed state, where the CS sits directly atop a MI pattern, we measure the time-resolved round-trip-by-round-trip dynamics of the intracavity field. In order to clearly distinguish between a $\mathrm{cw}$ background and a MI pattern (whose 160-GHz repetition rate is beyond the bandwidth of our detectors), we record the output field after a 1.6-nm (full width at half maximum) bandpass filter that is offset by about $1.5 \mathrm{~nm}$ from the pump wavelength. This removes the cw component at the pump wavelength, thus ensuring that all measured signals are purely borne of the MI and CS fields.

The density map in Fig. 3(a) shows a sequence of experimentally recorded oscilloscope traces, concatenated on top of each other so as to illustrate the round-trip-byround-trip evolution of the intracavity field. The measurement is taken immediately after the CS excitation process, 

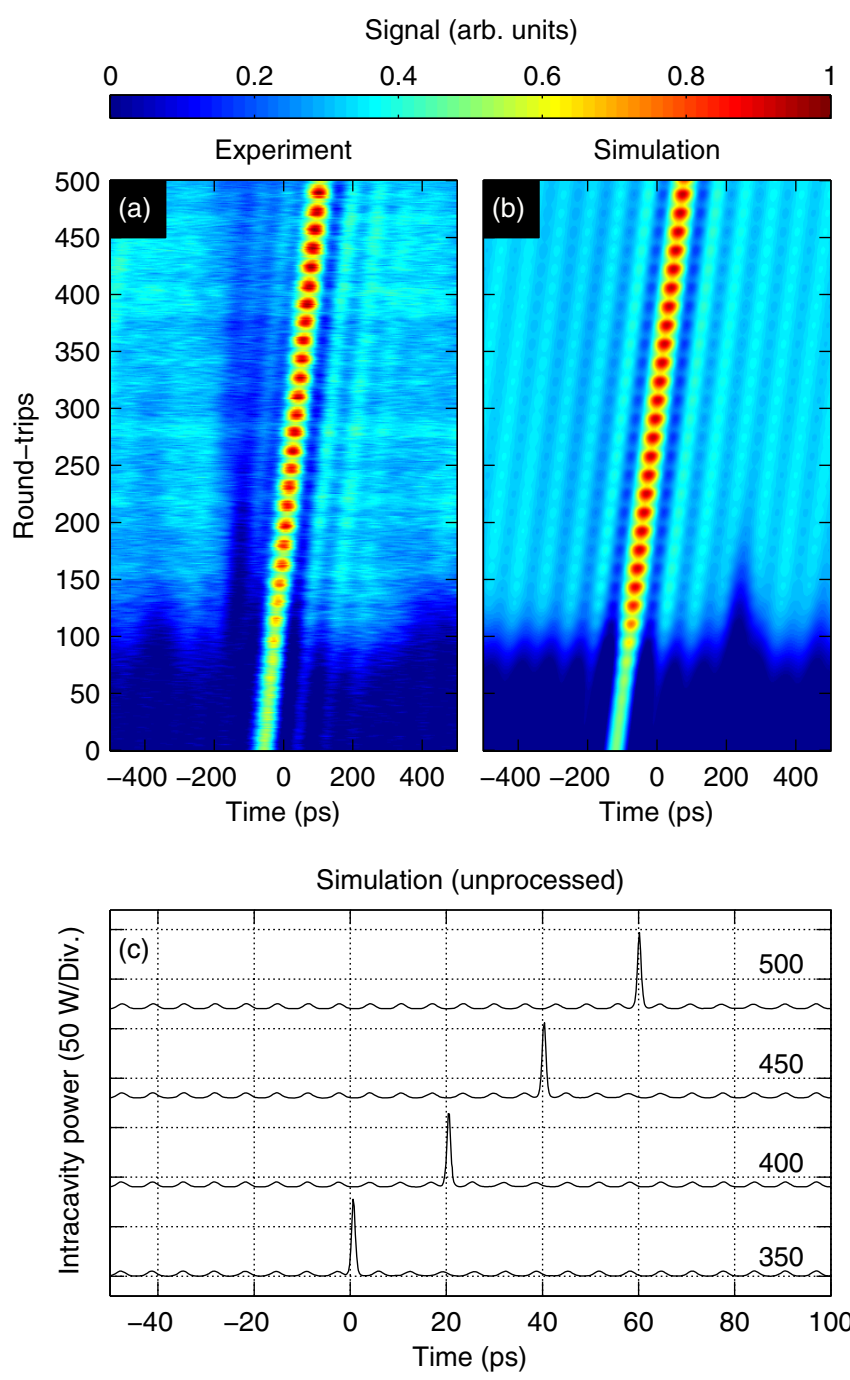

FIG. 3. Real-time dynamics of a CS on top of a patterned background. (a) Vertically concatenated segments extracted from a single oscilloscope trace measured at the cavity output after an offset filter, showing the round-trip-by-round-trip evolution of the intracavity field after the detuning has settled to a constant value. (b) Corresponding results from numerical simulations, processed to take into account the experimental detection method. The oscillatory features arise from the CS drifting across the MI pattern. (c) Temporal profiles at four different round-trips (indicated on the right) extracted from the simulation before taking into account the experimental detection method. The profiles are vertically offset for clarity. For a full animation of the simulation, see Supplemental Material [80].

when the detuning sweep has settled to the constant value of $\delta_{1}=1.79 \pi$, and it captures the nascent MI pattern (extended light blue trace) emerging from the cw background (zero signal, dark blue) that initially surrounds a single CS. It is worth highlighting that the weak temporal modulations (of the light blue background) around the measured CS signal do not correspond to the MI pattern. With a period of about $70 \mathrm{ps}$, these modulations simply stem from the impulse response of our 12.5-GHz detection system (photodetector and oscilloscope). The 160-GHz MI pattern cannot be directly resolved with our photodetector, and its emergence is rather evidenced by the increase in background signal level (recall that the measurement is taken after an offset filter that removes the cw component).

Surprisingly, as soon as the MI pattern emerges, the peak amplitude of the CS signal begins to noticeably oscillate with a period of about 16.1 round-trips. These oscillations (along with the other surrounding dynamics) are fully captured by numerical simulations of our experiment, as shown in Fig. 3(b). To better corroborate our experimental findings, the simulation results we show here are postprocessed to mimic our detection methods (i.e., the simulation takes into account the offset filter and the impulse response of our detection system). Closer analysis of the unprocessed data [cf. Fig. 3(c)] reveals that the oscillations in the peak amplitude of the CS signal originate from the group-velocity mismatch between the CS and the MI pattern, dominantly caused by the soliton's Raman selffrequency shift $[37,48,51,81]$. Specifically, SRS shifts the spectral center of the soliton towards longer wavelengths, which gives rise to a change in group velocity (see Appendix A); the oscillations in power measured through the offset filter arise as the CS drifts across the periodic MI pattern [see Fig. 3(c) and the animation in the Supplemental Material [80]]. Given that the temporal period of the measured pattern is about $6.3 \mathrm{ps}$, and that the oscillations have a constant period of 16.1 round-trips, we experimentally estimate a CS drift rate of $V=0.39 \mathrm{ps} /$ round-trip. This, in turn, corresponds to a Raman self-frequency shift of $\Delta f=V /\left(2 \pi \beta_{2}\right) \sim-27 \mathrm{GHz}$, in close agreement with the subtle $25 \mathrm{GHz}$ redshift inferred from the experimentally measured spectrum shown in Fig. 2(c). Both of these values are also in excellent agreement with those extracted from our simulations: $V_{\text {sim }} \sim 0.40 \mathrm{ps} /$ round-trip and $\Delta f_{\text {sim }} \sim-28 \mathrm{GHz}$. Note that, if we neglect SRS in our simulations (setting $f_{R}=0$ ), the oscillations in CS peak amplitude cease, further confirming that the behavior ensues from Raman-induced redshift.

The experimental results shown in Figs. 2 and 3 very clearly confirm that the states we observe correspond to a genuine mixed state where a CS is surrounded by an extended MI pattern, and this interpretation is fully corroborated by our numerical simulations. In addition to representing the first experimental observation of such a state, it is also worth emphasizing that, to the best of our knowledge, the coexistence of MI patterns and CSs associated with adjacent resonances has not even been theoretically proposed before.

\section{B. Coexistence of distinct CS states}

The 100-m-long cavity, used in the experiments we describe above, does not easily permit nonlinear phase shifts sufficient for two adjacent resonances to simultaneously support temporal CSs. To access that regime, we 
use our 300-m-long, high-finesse cavity. We set the peak power of the flattop driving pulses to about $2.6 \mathrm{~W}$, which generates a Kerr tilt of $3.7 \pi$. For these parameters, the cavity is predicted to exhibit $\mathrm{cw}$ tristability for detunings $\delta_{2}>0.22 \pi\left(\delta_{1}>2.22 \pi\right)$ measured from the second (first) resonance. Note that this tristability is not directly observable (as, e.g., characteristic hysteresis behavior), as the $\mathrm{cw}$ states are stable only against homogeneous $(\mathrm{cw})$ perturbations; the unavoidable MI of the two upper states hides direct signatures. Of course, the presence of two modulationally unstable (and one unconditionally stable) cw states underpins the coexistence of two distinct CS states [see Fig. 1(a)], which therefore acts as a convincing signature of tristability.

Numerical simulations of the Ikeda map show that, for large detunings, the direct excitation $[30,82]$ of a "super" CS is extremely difficult, requiring a very carefully shaped initial condition. As it is not feasible to experimentally tailor a perturbation with sufficient precision, we rely on the spontaneous excitation and adiabatic transformation of CSs as the cavity detuning is continuously increased [34,49]. Specifically, to reach an intracavity state consisting of two distinct CSs atop a cw background [as in Fig. 1(e)], we scan the frequency of the pump laser across two cavity resonances. To facilitate the interpretation of our experimental data, we first describe results obtained from corresponding numerical simulations of Eqs. (1) and (2). For these simulations, we set the initial detuning to $\delta_{0}=-0.2 \pi$, continuously increase it to $\delta_{0}=2.7 \pi$ over 350 round-trips, then subsequently maintain it at this level for 100 further round-trips. Results are shown in Fig. 4, where we plot the simulated evolution of the intracavity intensity over a 500-ps time window as a function of round-trip number and detuning.

In our simulation, we first see the formation of a MI pattern across the entire cavity [round-trips 50-85, labeled "MI 1" in Fig. 4(a)] as we scan along the first resonance. As expected based on earlier studies [60], the pattern is initially stable, but then transforms into an unstable state consisting of fluctuating structures. Around the 100th round-trip, a sequence of localized temporal CSs is seen to emerge from the unstable MI state, evolving freely until the detuning reaches the second resonance [round-trips 85-270, "CS 1" in Fig. 4(a)]. The CSs occasionally collide with one another, which leads to merging or annihilation depending on the precise detuning at the round-trip of collision $[49,64]$. We can also see how the CSs exhibit markedly curved trajectories. This is due to the combined effect of SRS, GVD, and the continuously increasing detuning. Specifically, the CS drift rate is proportional to the Raman-induced redshift $\left(V \propto \beta_{2} \Delta f\right)$, which has been shown to be quadratically proportional to the detuning $\left(\Delta f \propto \delta_{0}^{2}\right)$ [81]. At round-trip 270 , the driving laser passes the MI threshold of the second resonance, and we can indeed see the emergence of a periodic pattern that coexists with the CSs from the first
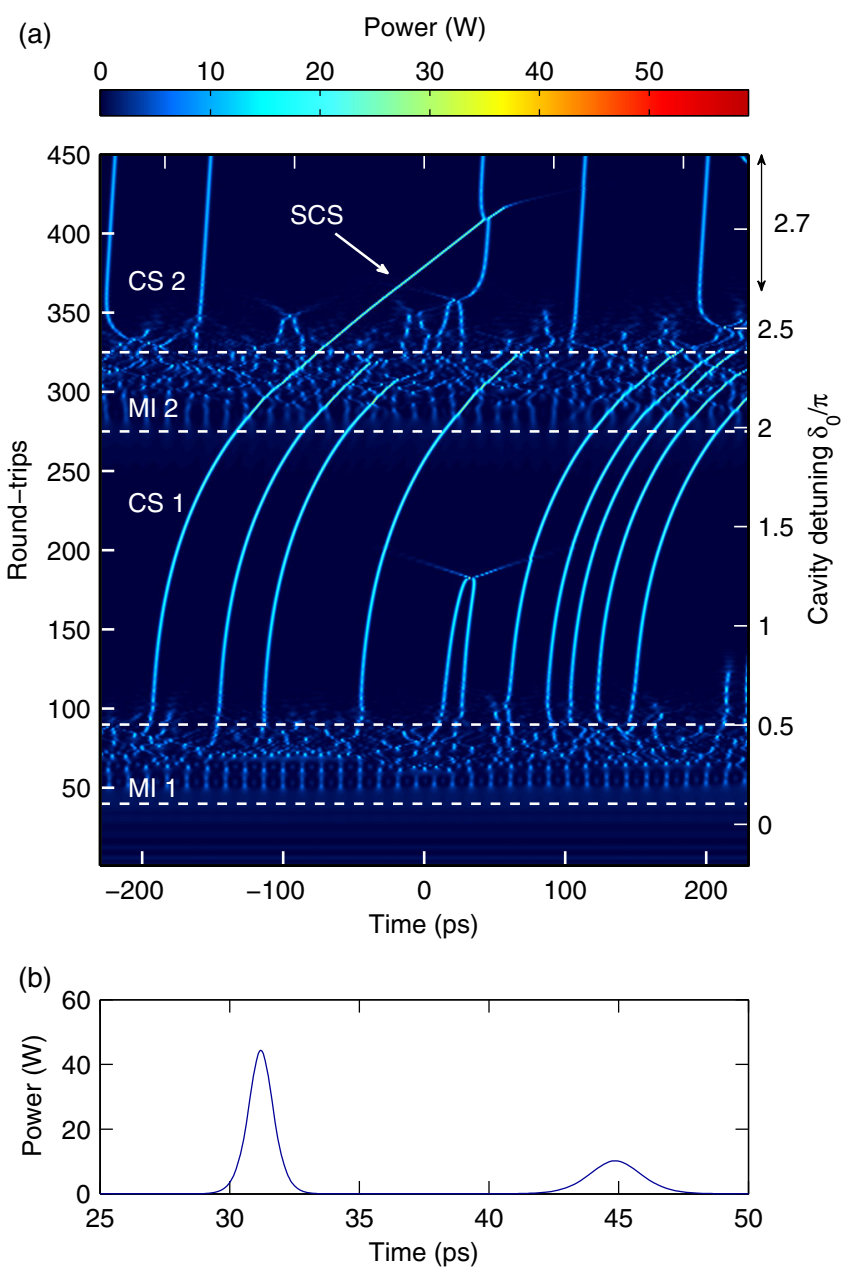

FIG. 4. (a) Numerical simulation of the Ikeda map, showing the intracavity dynamics as the cavity detuning is scanned across two cavity resonances and then maintained at $\delta_{0}=2.7 \pi$. Dashed horizontal lines highlight the boundaries of different dynamical regimes, as indicated. (b) Simulated temporal profile at roundtrip 400, before the collision of the two CSs associated with different resonances. SCS is the "super" CS associated with the first resonance. For a full animation of the simulation, see Supplemental Material [83].

resonance ["MI 2" in Fig. 4(a)]. As before, the MI pattern is initially stable but develops strong round-trip-to-round-trip fluctuations for larger detunings.

Our simulations show that the CSs emerging from the first resonance have a high likelihood of disappearing in the unstable MI region of the second resonance. More detailed analysis reveals that, although CSs can stably coexist with stable MI patterns, they tend to annihilate in the unstable MI regime as they collide with sufficiently large background fluctuations. In the simulation shown in Fig. 4(a), only a single temporal CS (highlighted by a white arrow) survives the MI region, persisting as the detuning increases to the regime of $\mathrm{cw}$ tristability. In that region, the CS from the first resonance coexists with those reshaping from the unstable MI pattern associated with the second resonance; 
the former now corresponds to a "super" CS as defined in Ref. [61]. Because this "super" CS is associated with a much larger detuning than those associated with the second resonance, it possesses a significantly shorter duration [as seen in Fig. 4(b)], and as a result, a more pronounced Raman-induced redshift (see also Appendix A). This difference in central wavelengths is evident based on the visibly different group velocities. [We emphasize that the soliton trajectories are linear (instead of curved) in the regime of CS coexistence, as the detuning is maintained at a constant value in this region.] Because of their different velocities, the "super" CS eventually collides with a soliton associated with the second resonance, and is annihilated due to the perturbation suffered.

In the experimental measurements corresponding to simulations shown in Fig. 4, we monitor the round-tripby-round-trip evolution of the intracavity field as we scan the pump laser across consecutive resonances. Specifically, we record the output of the 99/1 tap coupler using two 12.5-GHz photodetectors that are simultaneously sampled by the $40 \mathrm{GSa} / \mathrm{s}$ real-time oscilloscope: the first photodiode detects the output field directly, thus recording a signal proportional to the energy of each CS, while the second photodiode measures the output after it has been spectrally filtered by a 1-nm optical bandpass filter offset by $2 \mathrm{~nm}$ from the driving laser. Because the spectral width of a CS associated with the first (second) resonance is about $2 \mathrm{~nm}$ $(1 \mathrm{~nm})$ in the regime where they coexist, our second detection channel responds primarily to the presence of the solitons associated with the first resonance (i.e., the "super" CSs). This allows us to unambiguously discriminate experimentally between the two types of nonlinear structures.

In Figs. 5(a) and 5(b), we show oscilloscope traces recorded by our direct and offset filtered detection channels, respectively. As in our simulations above, the cavity detuning continuously increases until round-trip 350, after which we allow the system to evolve freely (i.e., without changing or locking the pump cavity detuning [84]). Despite the limited temporal resolution of our detection system, the experimental oscilloscope traces show all the features predicted in the numerical simulations. Indeed, the intracavity field first corresponds to an unstable MI state, out of which emerges a sequence of CSs that display noticeably curved time-domain trajectories. When the detuning increases beyond the MI threshold of the second resonance (around round-trip 270), a new (unstable) MI state arises and wipes out most of the CSs associated with the first resonance. However, as highlighted by the white arrow in Fig. 5(a), a single CS survives the MI region unscathed, coexisting with a sequence of newly formed solitons associated with the second resonance. We can indeed easily distinguish between the two types of CS states based on two clear experimental observations. First, the solitons clearly exhibit different drift velocities: the lone soliton of the first resonance follows the trajectory it had prior to the MI region,

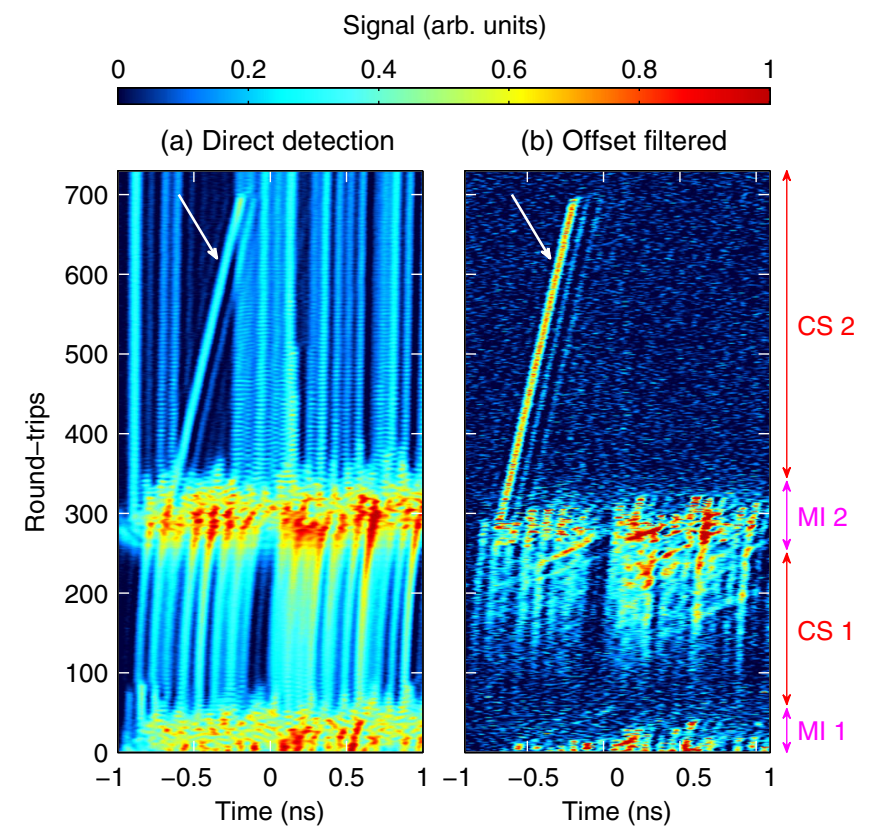

FIG. 5. Experimental results, showing evidence of the coexistence of two different CS states. (a), (b) Vertically concatenated segments of oscilloscope traces measured as the driving laser frequency is first scanned across two cavity resonances (roundtrips 1-350) and then maintained at a constant value (round-trips 351-750). Traces in (a) were measured directly at the cavity output while those in (b) were obtained after the output had passed through an offset filter. The different slopes and spectral bandwidths of the localized structures emerging around the 350th round-trip are indicative of two different CS states. The white arrow highlights a "super" CS associated with the first resonance.

while the curvatures of the newly formed solitons' trajectories are reset. [Note that, as in Fig. 4, the detuning does not change for round-trips beyond 350, explaining the linear (rather than curved) trajectories.] Second, only the soliton of the first resonance appears in the trace measured in our offset-filtered detection channel [see Fig. 5(b)], evidencing its much larger spectral width compared to the other solitons. Taken together, these results reveal unequivocally that we observe the coexistence of different CS states associated with adjacent resonances. Similarly to our numerical simulations, our experiments also show clear evidence (around round-trip 680) of the "super" CS of the first resonance being annihilated after it collides with a soliton associated with the second resonance.

We must note that scanning into the regime of CS coexistence is a probabilistic process, as solitons from the first resonance can annihilate when crossing the unstable MI regime of the second resonance. Intuitively, the number of solitons surviving the MI regime (per scan realization) increases with the temporal duration of the quasi-cw pump pulses (as more solitons can be sustained overall) and the rate of detuning scan (as less time is spent in the unstable MI regime). We confirm these dependencies by means of 
extensive Monte Carlo simulations (for details, see Supplemental Material [79]). Because of the difficulty of actively stabilizing our 300-m-long cavity, experimental analysis of the statistics is intractable using our current setup. Nevertheless, we emphasize that the results presented in Fig. 5 are highly repeatable: only a few minutes of manual data accumulation and analysis in the laboratory is enough for a positive observation of a long-lasting "super" CS. In our fiber-based experiment (that is influenced by SRS), the lifetimes of the "super" CSs are also probabilistic: the number of round-trips before a collision occurs depends on the (random) configuration of solitons after the chaotic MI stage. Although the lifetimes are typically measured in tens of round-trips, outliers persisting for hundreds of round-trips (as in Figs. 4 and 5) are not infrequent (for statistics, see Supplemental Material [79]).

\section{DISCUSSION AND CONCLUSIONS}

We report the first combined experimental and theoretical study of Kerr cavity dynamics in the strongly nonlinear regime, where adjacent cavity resonances overlap. We demonstrate how new combinations of nonlinear states may emerge in this regime, and how they can be understood as mixed states composed of structures associated with individual resonances. To the best of our knowledge, we present the first experimental demonstration of the stable coexistence between a temporal CS and a MI pattern, and report the first experimental signatures of the coexistence and interactions between two distinct CS states. In this way, our work directly confirms the theoretically predicted existence of "super" CSs [61]. More generally, however, our theoretical and experimental results show that the coexistence of nonlinear structures is not limited to CSs, but encompasses arbitrary combinations of structures associated with adjacent resonances, including periodic patterned states (both stable and unstable).

Our work raises a number of interesting questions for follow-up research: what are the (nonlinear) dynamics of coexisting CSs and MI patterns in the absence of Raman scattering; how are the dynamics stemming from overlapping resonances related to dynamics of dual-pumped Kerr cavities; is it possible to systematically control the excitation probability and lifetimes of "super" CSs in realistic systems? Furthermore, we expect that our general findings will resonate beyond passive Kerr cavities, and that novel mixed states await discovery in other dissipative systems exhibiting tilted homogeneous solutions, such as, e.g., quadratically nonlinear optical resonators $[85,86]$. By identifying and experimentally confirming the general concept of coexisting nonlinear states, our work paves the way for the future study of such dynamics.

Before closing, we briefly comment on the relevance of our findings to studies of microresonator frequency combs that are currently under intense investigations. Assuming critical coupling and high cavity finesse, the maximum nonlinear phase shift of a cw state can be approximated as $\phi_{\mathrm{NL}} \approx \gamma P_{\text {in }} L \mathcal{F} / \pi$. While $\phi_{\mathrm{NL}} \ll 2 \pi$ in most microresonator studies, strong nonlinearities (and hence large nonlinear phase shifts) are required for the generation of ultrabroadband frequency combs. Indeed, a Kerr tilt of about $0.7 \pi$ was reached when generating an octave-spanning comb in a 40 - $\mu$ m-radius silica microtoroid $\left(\mathcal{F} \approx 1.2 \times 10^{6}\right)$ driven with $P_{\text {in }} \approx 1 \mathrm{~W}$ [62]. To our knowledge, this represents the largest Kerr nonlinear phase shift reported to date in a microresonator experiment, yet we envisage that the continuous push towards broader spectral bandwidths could ultimately propel such systems into the highly nonlinear regime with $\phi_{\mathrm{NL}} \gtrsim 2 \pi$. More immediately, by showing that the LLE can be safely applied to predict CS characteristics even when the condition $\delta_{0} \ll 1$ is not strictly satisfied, our work consolidates the equations' broad applicability to situations that are of more immediate relevance to current-day microresonator frequency comb experiments (with $\delta_{0} \sim 1$ ). Finally, although we focus on resonances that overlap due to Kerr nonlinear phase shifts, we speculate that other mechanisms (e.g., interactions between different mode families) could engender similar overlap even for $\phi_{\mathrm{NL}} \ll 2 \pi$. This speculation is corroborated by recent experimental observations of microresonator frequency combs that show qualitative signatures of coexisting nonlinear states [87]. Our work demonstrates that such structures can physically arise due to the overlap of adjacent resonances, and could thus represent a key step towards explaining the particular frequency comb structures observed.

\section{ACKNOWLEDGMENTS}

We acknowledge support from the Marsden Fund and the Rutherford Discovery Fellowships of the Royal Society of New Zealand. We are grateful for useful discussions with Gian-Luca Oppo, Fabio Biancalana, Matteo Conforti, and Thorsten Ackemann.

\section{APPENDIX A: STIMULATED RAMAN SCATTERING}

In the context of Kerr cavity dynamics studied in our work, stimulated Raman scattering dominantly manifests itself by shifting the spectral center of mass of MI patterns and temporal CSs towards longer wavelengths through the so-called intrapulse Raman scattering [37,48,51]. Similarly to soliton self-frequency shift in optical fiber [88], the magnitude of the frequency shift scales as $\Delta f \propto \Delta \tau^{-4}$, where $\Delta \tau$ is the temporal duration of the nonlinear structure [89]. Compounded by the fact that the duration of CSs scales as $\delta_{0}^{-1 / 2}$, the effect can thus be expected to be particularly important for CSs at high detunings, with $\Delta f \propto$ $\delta_{0}^{2}$ [81]. Because of dispersion, the frequency shift also gives rise to change in group velocity, resulting in a temporal drift with respect to the reference frame moving 
at the velocity of light of the pump wavelength [81]. Specifically, over a single round-trip, a spectral shift $\Delta f$ will result in the accrual of an extra group delay given by $V=2 \pi \Delta f \beta_{2} L$.

Because, for constant parameters, different nonlinear structures are associated with different temporal durations [see, e.g., Fig. 1(e)], they may experience different degrees of Raman self-frequency shift. As shown in Figs. 3 and 5, the resulting group-velocity variations result in identifiable signatures in real-time measurements, allowing us to reliably discriminate between different nonlinear states.

It is important to emphasize that, in a dissipative cavity system with constant parameters, the nonlinear structures perturbed by SRS typically reach steady state in the sense that the spectral shift $\Delta f$ and corresponding (per roundtrip) relative group delay $V$ remain constant from round-trip to round-trip [48]. This should be contrasted with the dynamics of conventional (conservative) solitons in singlepass fiber or waveguide systems, namely, continuous spectral redshift (accompanied by temporal deceleration) along propagation [68]. In this context, we emphasize that the curved temporal trajectories observed in Figs. 4 and 5 are simply manifestations of the (adiabatically) increasing detuning $\left(V \propto \Delta f \propto \delta_{0}^{2}\right.$ ), and should not be confused with deceleration in single-pass systems with constant parameters. Finally, we note that these curved trajectories agree quantitatively with the trajectories expected to arise from the interplay of Raman redshift, group-velocity dispersion, and a continuously increasing detuning [81].

\section{APPENDIX B: MEAN-FIELD APPROACH}

If the slowly varying field envelope $E(z, \tau)$ does not change significantly as it propagates once around the fiber loop [such that $E(z, \tau) \approx E(0, \tau)$ on the right-hand side of Eq. (2)], and if the cavity detuning and losses are both small $\left(\delta_{0}, \rho \ll 1\right)$, the Ikeda map [Eqs. (1) and Eqs. (2)] can be averaged into a single externally driven nonlinear Schrödinger equation $[35,48,51,63]$ :

$$
\begin{aligned}
t_{R} \frac{\partial E(t, \tau)}{\partial t}= & {\left[-\alpha-i \delta_{0}-\frac{i L \beta_{2}}{2} \frac{\partial^{2}}{\partial \tau^{2}}\right] E+\sqrt{\theta} E_{\mathrm{in}} } \\
& +i \gamma L\left[R(\tau) *|E(\tau)|^{2}\right] E
\end{aligned}
$$

where $\alpha \approx(1-\rho) / 2$. In the absence of the Raman nonlinearity $\left(f_{R}=0\right)$, Eq. (B1) is fully analogous to the LugiatoLefever equation of spatially diffractive Kerr cavities [10].

Because the standard derivation of Eq. (B1) assumes $\delta_{0} \ll 1$ [63], it is not particularly surprising that the equation cannot describe the mixed states involving several adjacent resonances with $\delta_{0} \approx 2 \pi$ (see also the discussion on $\mathrm{cw}$ solutions in Appendix C). However, as shown in Fig. 1(e), we generically find that the individual nonlinear states (CSs, patterns) that make up the mixed states are quite well reproduced by Eq. (B1), provided that the cavity detuning $\delta_{0}$ is quoted relative to the appropriate resonance (and that the cavity has high finesse so as to ensure that the intracavity field evolves only slightly over one round-trip). This result is somewhat surprising, as it demonstrates the ability of the mean-field Eq. (B1) to accurately predict the characteristics of CSs even at very large detunings, $\delta_{0} \gg 1$. In this context, we emphasize that, although the CS branches shown in Fig. 1(a) are obtained by applying an iterative Newton-Raphson algorithm [35] on Eq. (B1), full simulations of the Ikeda map predict similar ranges of CS existence (with discrepancies arising mostly from the finite finesse).

Finally, we note briefly that Kartashov et al. have very recently proposed a generalized LLE [90], where the driving term is localized around a point rather than distributed around the whole ring resonator [as in Eq. (B1)]. Furthermore, Conforti and Biancalana have proposed a set of coupled LLEs to describe interactions between adjacent cavity modes [91]. Similarly to the Ikeda map, both of these approaches appear to allow for the full description of coexisting nonlinear states.

\section{APPENDIX C: cw STEADY-STATE SOLUTIONS}

The cw $\left[\partial E_{m}(z, \tau) / \partial \tau=0\right]$ steady-state $\left[E_{m+1}(z=0)=\right.$ $E_{m}(z=0)$ ] solutions of the Ikeda map [Eqs. (1) and (2)] satisfy the familiar Airy equation of a nonlinear FabryPérot resonator,

$$
P=\frac{\theta P_{\text {in }}}{(1-\sqrt{\rho})^{2}\left[1+F \sin ^{2}\left(\frac{\delta_{0}-\gamma L P}{2}\right)\right]},
$$

where $P=\left|E_{m}(z=0)\right|^{2}$ and $P_{\text {in }}=\left|E_{\text {in }}\right|^{2}$ correspond to power levels of the intracavity and the driving fields, respectively, and $F=4 \sqrt{\rho} /(1-\sqrt{\rho})^{2}$. The solutions of Eq. (C1) describe the periodically repeating, tilted cavity resonances, whose peaks are nonlinearly displaced by $\phi_{\mathrm{NL}}=$ $\gamma L P_{\max }$, where $P_{\max }=\theta P_{\text {in }} /(1-\sqrt{\rho})^{1 / 2} \approx \theta P_{\text {in }} \mathcal{F}^{2} / \pi^{2}$ is the peak intracavity power. When the maximum phase displacement $\phi_{\mathrm{NL}}$ is larger than the resonance width $\Delta \phi=2 \pi / \mathcal{F}$, the cw response becomes multivalued and exhibits the well-known hysteresis of dispersive optical bistability. In the more extreme situation, where $\phi_{\mathrm{NL}}>2 \pi$ [as in Fig. 1(a)], adjacent resonances actually overlap. In addition to bistability associated with individual resonances, such a large nonlinear tilt can give rise to regions of $\mathrm{cw}$ tristability, i.e., regions where the system has three homogeneous equilibrium points that are stable against $\mathrm{cw}$ perturbations [61].

In the mean-field limit, where the Kerr cavity dynamics are described by Eq. (B1), the cw steady-state solutions satisfy the well-known cubic polynomial:

$$
\theta P_{\text {in }}=(\gamma L)^{2} P^{3}-2 \delta_{0} \gamma L P^{2}+\left(\alpha^{2}+\delta_{0}^{2}\right) P .
$$

In contrast to Eq. (C1), the solutions of Eq. (C2) describe a single nonlinearly tilted Lorentzian resonance. To illustrate 


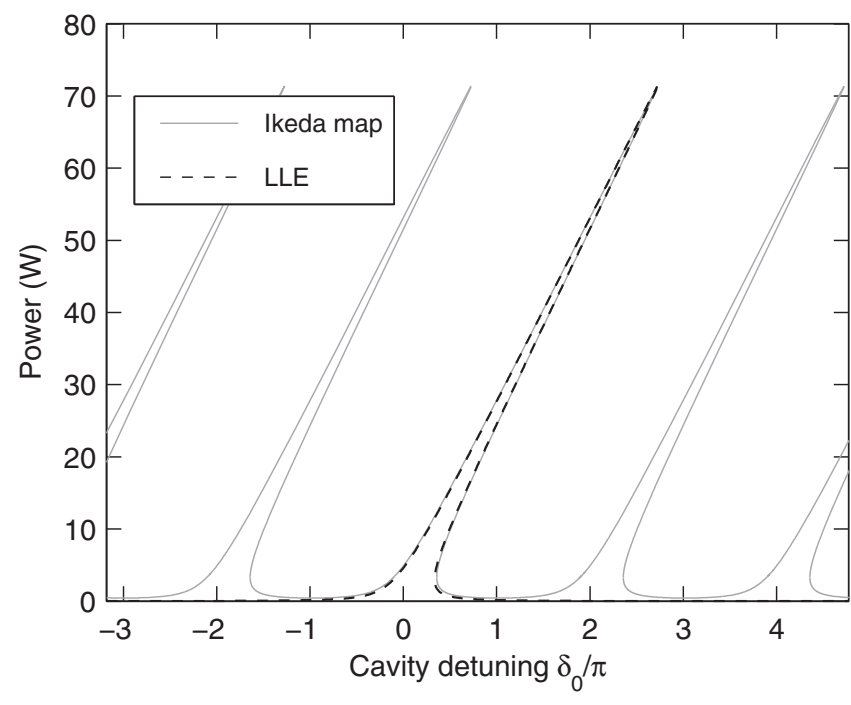

FIG. 6. Comparison of cw solutions of the Ikeda map (solid gray curves) and the LLE (dashed black curves). All parameters the same as in Fig. 1.

the difference, in Fig. 6 we compare the cw solutions obtained from the two different models (parameters as in Fig. 1). As can be seen, the cw solutions obtained from Eq. (C2) (black dashed lines) agree very well with a single cycle of the periodically repeating resonances predicted by Eq. (C1) (gray solid curves), but decay to zero as $\delta_{0} \rightarrow \pm \infty$. This should make clear why the mean-field Eq. (B1) is unable to describe mixed states consisting of structures associated with adjacent resonances.

[1] A. Szöke, V. Daneu, J. Goldhar, and N. A. Kurnit, Bistable Optical Element and Its Applications, Appl. Phys. Lett. 15, 376 (1969).

[2] G. S. McDonald and W. J. Firth, Spatial Solitary-Wave Optical Memory, J. Opt. Soc. Am. B 7, 1328 (1990).

[3] S. Wabnitz, Suppression of Interactions in a Phase-Locked Soliton Optical Memory, Opt. Lett. 18, 601 (1993).

[4] S. Barland, J. R. Tredicce, M. Brambilla, L. A. Lugiato, S. Balle, M. Giudici, T. Maggipinto, L. Spinelli, G. Tissoni, T. Knödl, M. Miller, and R. Jäger, Cavity Solitons as Pixels in Semiconductor Microcavities, Nature (London) 419, 699 (2002).

[5] J. K. Jang, M. Erkintalo, J. Schröder, B. J. Eggleton, S. G. Murdoch, and S. Coen, All-Optical Buffer Based on Temporal Cavity Solitons Operating at $10 \mathrm{~Gb} / \mathrm{s}$, Opt. Lett. 41, 4526 (2016).

[6] S. D. Smith, Optical Bistability: Towards the Optical Computer, Nature (London) 307, 315 (1984).

[7] Q. Vinckier, F. Duport, A. Smerieri, K. Vandoorne, P. Bienstman, M. Haelterman, and S. Massar, HighPerformance Photonic Reservoir Computer Based on a Coherently Driven Passive Cavity, Optica 2, 438 (2015).
[8] L. Lugiato, F. Prati, and M. Brambilla, Nonlinear Optical Systems (Cambridge University Press, Cambridge, England, 2015).

[9] D. W. M. Laughlin, J. V. Moloney, and A. C. Newell, Solitary Waves as Fixed Points of Infinite-Dimensional Maps in an Optical Bistable Ring Cavity, Phys. Rev. Lett. 51, 75 (1983).

[10] L. A. Lugiato and R. Lefever, Spatial Dissipative Structures in Passive Optical Systems, Phys. Rev. Lett. 58, 2209 (1987).

[11] G.-L. Oppo, M. Brambilla, and L. A. Lugiato, Formation and Evolution of Roll Patterns in Optical Parametric Oscillators, Phys. Rev. A 49, 2028 (1994).

[12] F. T. Arecchi, S. Boccaletti, and P. Ramazza, Pattern Formation and Competition in Nonlinear Optics, Phys. Rep. 318, 1 (1999).

[13] N. N. Rosanov, Spatial Hysteresis and Optical Patterns (Springer, Berlin, 2002).

[14] W. J. Firth, in Soliton-Driven Photonics, edited by A. D. Boardman and A.P. Sukhorukov (Springer, Netherlands, 2001).

[15] T. Ackemann and W. Firth, in Dissipative Solitons, Lecture Notes in Physics, edited by N. Akhmediev and A. Ankiewicz (Springer, Berlin, 2005).

[16] T. Ackemann, W. Firth, and G. Oppo, Fundamentals and Applications of Spatial Dissipative Solitons in Photonic Devices, Adv. At. Mol. Opt. Phys. 57, 323 (2009).

[17] M. Tlidi, M. Taki, and T. Kolokolnikov, Introduction: Dissipative Localized Structures in Extended Systems, Chaos 17, 037101 (2007).

[18] W. J. Firth and A. J. Scroggie, Optical Bullet Holes: Robust Controllable Localized States of a Nonlinear Cavity, Phys. Rev. Lett. 76, 1623 (1996).

[19] C. Etrich, U. Peschel, and F. Lederer, Solitary Waves in Quadratically Nonlinear Resonators, Phys. Rev. Lett. 79, 2454 (1997).

[20] M. Marconi, J. Javaloyes, S. Barland, S. Balle, and M. Giudici, Vectorial Dissipative Solitons in Vertical-Cavity Surface-Emitting Lasers with Delays, Nat. Photonics 9, 450 (2015).

[21] K. Ikeda, H. Daido, and O. Akimoto, Optical Turbulence: Chaotic Behavior of Transmitted Light from a Ring Cavity, Phys. Rev. Lett. 45, 709 (1980).

[22] H. J. Carmichael, in Proceedings of the Third New Zealand Symposium on Laser Physics, Hamilton, New Zealand, 1983, edited by J. D. Harvey and D. F. Walls (Springer, Berlin, 1983), pp. 17-23.

[23] D. W. McLaughlin, J. V. Moloney, and A.C. Newell, New Class of Instabilities in Passive Optical Cavities, Phys. Rev. Lett. 54, 681 (1985).

[24] J. Garca-Ojalvo and R. Roy, Spatiotemporal Communication with Synchronized Optical Chaos, Phys. Rev. Lett. 86, 5204 (2001).

[25] P. Coullet, L. Gil, and F. Rocca, Optical Vortices, Opt. Commun. 73, 403 (1989).

[26] J. Scheuer and M. Orenstein, Optical Vortices Crystals: Spontaneous Generation in Nonlinear Semiconductor Microcavities, Science 285, 230 (1999).

[27] C. J. Gibson, A. M. Yao, and G.-L. Oppo, Optical Rogue Waves in Vortex Turbulence, Phys. Rev. Lett. 116, 043903 (2016). 
[28] B. Garbin, J. Javaloyes, G. Tissoni, and S. Barland, Topological Solitons as Addressable Phase Bits in a Driven Laser, Nat. Commun. 6, 5915 (2015).

[29] S. Coen and M. Erkintalo, in Nonlinear Optical Cavity Dynamics, edited by $\mathrm{Ph}$. Grelu (Wiley-VCH, Weinheim, Germany, 2016).

[30] F. Leo, S. Coen, P. Kockaert, S.-P. Gorza, Ph. Emplit, and M. Haelterman, Temporal Cavity Solitons in OneDimensional Kerr Media as Bits in an All-Optical Buffer, Nat. Photonics 4, 471 (2010).

[31] F. Leo, L. Gelens, Ph. Emplit, M. Haelterman, and S. Coen, Dynamics of One-Dimensional Kerr Cavity Solitons, Opt. Express 21, 9180 (2013).

[32] J. K. Jang, M. Erkintalo, S. G. Murdoch, and S. Coen, Ultraweak Long-Range Interactions of Solitons Observed Over Astronomical Distances, Nat. Photonics 7, 657 (2013).

[33] J. K. Jang, M. Erkintalo, S. Coen, and S. G. Murdoch, Temporal Tweezing of Light through the Trapping and Manipulation of Temporal Cavity Solitons, Nat. Commun. 6, 7370 (2015).

[34] T. Herr, V. Brasch, J. D. Jost, C. Y. Wang, N. M. Kondratiev, M. L. Gorodetsky, and T. J. Kippenberg, Temporal Solitons in Optical Microresonators, Nat. Photonics 8, 145 (2014).

[35] S. Coen, H. G. Randle, T. Sylvestre, and M. Erkintalo, Modeling of Octave-Spanning Kerr Frequency Combs Using a Generalized Mean-Field Lugiato-Lefever Model, Opt. Lett. 38, 37 (2013).

[36] Y. K. Chembo and C. R. Menyuk, Spatiotemporal LugiatoLefever Formalism for Kerr-Comb Generation in Whispering-Gallery-Mode Resonators, Phys. Rev. A 87, 053852 (2013).

[37] X. Yi, Q.-F. Yang, K. Y. Yang, M.-G. Suh, and K. Vahala, Soliton Frequency Comb at Microwave Rates in a High- $Q$ Silica Microresonator, Optica 2, 1078 (2015).

[38] V. Brasch, M. Geiselmann, T. Herr, G. Lihachev, M. H. P. Pfeiffer, M. L. Gorodetsky, and T. J. Kippenberg, Photonic Chip-Based Optical Frequency Comb Using Soliton Cherenkov Radiation, Science 351, 357 (2016).

[39] C. Joshi, J. K. Jang, K. Luke, X. Ji, S. A. Miller, A. Klenner, Y. Okawachi, M. Lipson, and A. L. Gaeta, Thermally Controlled Comb Generation and Soliton Modelocking in Microresonators, Opt. Lett. 41, 2565 (2016).

[40] K. E. Webb, M. Erkintalo, S. Coen, and S. G. Murdoch, Experimental Observation of Coherent Cavity Soliton Frequency Combs in Silica Microspheres, Opt. Lett. 41, 4613 (2016).

[41] T. J. Kippenberg, R. Holzwarth, and S. A. Diddams, Microresonator-Based Optical Frequency Combs, Science 332, 555 (2011).

[42] J. Pfeifle, V. Brasch, M. Lauermann, Y. Yu, D. Wegner, T. Herr, K. Hartinger, P. Schindler, J. Li, D. Hillerkuss, R. Schmogrow, C. Weimann, R. Holzwarth, W. Freude, J. Leuthold, T. J. Kippenberg, and C. Koos, Coherent Terabit Communications with Microresonator Kerr Frequency Combs, Nat. Photonics 8, 375 (2014).

[43] J. Pfeifle, A. Coillet, R. Henriet, K. Saleh, P. Schindler, C. Weimann, W. Freude, I. V. Balakireva, L. Larger, C. Koos, and Y. K. Chembo, Optimally Coherent Kerr Combs Generated with Crystalline Whispering Gallery Mode
Resonators for Ultrahigh Capacity Fiber Communications, Phys. Rev. Lett. 114, 093902 (2015).

[44] M.-G. Suh, Q.-F. Yang, K. Y. Yang, X. Yi, and K. J. Vahala, Microresonator Soliton Dual-Comb Spectroscopy, Science 354, 600 (2016).

[45] A. Dutt, C. Joshi, X. Ji, J. Cardenas, Y. Okawachi, K. Luke, A. L. Gaeta, and M. Lipson, On-Chip Dual Comb Source for Spectroscopy, arXiv:1611.07673.

[46] C. Milián and D. V. Skryabin, Soliton Families and Resonant Radiation in a Micro-Ring Resonator Near Zero Group-Velocity Dispersion, Opt. Express 22, 3732 (2014).

[47] J. K. Jang, M. Erkintalo, S. G. Murdoch, and S. Coen, Observation of Dispersive Wave Emission by Temporal Cavity Solitons, Opt. Lett. 39, 5503 (2014).

[48] C. Milián, A. V. Gorbach, M. Taki, A. V. Yulin, and D. V. Skryabin, Solitons and Frequency Combs in Silica Microring Resonators: Interplay of the Raman and Higher-Order Dispersion Effects, Phys. Rev. A 92, 033851 (2015).

[49] K. Luo, J. K. Jang, S. Coen, S. G. Murdoch, and M. Erkintalo, Spontaneous Creation and Annihilation of Temporal Cavity Solitons in a Coherently Driven Passive Fiber Resonator, Opt. Lett. 40, 3735 (2015).

[50] H. Guo, M. Karpov, E. Lucas, A. Kordts, M. H. P. Pfeiffer, V. Brasch, G. Lihachev, V. E. Lobanov, M. L. Gorodetsky, and T. J. Kippenberg, Universal Dynamics and Deterministic Switching of Dissipative Kerr Solitons in Optical Microresonators, Nat. Phys. 13, 94 (2017).

[51] M. Karpov, H. Guo, A. Kordts, V. Brasch, M. H. Pfeiffer, M. Zervas, M. Geiselmann, and T. J. Kippenberg, Raman SelfFrequency Shift of Dissipative Kerr Solitons in an Optical Microresonator, Phys. Rev. Lett. 116, 103902 (2016).

[52] Q.-F. Yang, X. Yi, K. Y. Yang, and K. Vahala, Stokes Solitons in Optical Microcavities, Nat. Phys. 13, 53 (2017).

[53] D. C. Cole, E. S. Lamb, P. Del'Haye, S. A. Diddams, and S. B. Papp, Soliton Crystals in Kerr Resonators, arXiv:1610 .00080 .

[54] M. Anderson, F. Leo, S. Coen, M. Erkintalo, and S. G. Murdoch, Observations of Spatiotemporal Instabilities of Temporal Cavity Solitons, Optica 3, 1071 (2016).

[55] C. Bao, J. A. Jaramillo-Villegas, Y. Xuan, D. E. Leaird, M. Qi, and A. M. Weiner, Observation of Fermi-Pasta-Ulam Recurrence Induced by Breather Solitons in an Optical Microresonator, Phys. Rev. Lett. 117, 163901 (2016).

[56] M. Yu, J. K. Jang, Y. Okawachi, A. G. Griffith, K. Luke, S. A. Miller, X. Ji, M. Lipson, and A. L. Gaeta, Breather Soliton Dynamics in Microresonators, Nat. Commun. 8, 14569 (2017).

[57] E. Lucas, M. Karpov, H. Guo, M. Gorodetsky, and T. Kippenberg, Breathing Dissipative Solitons in Optical Microresonators, arXiv:1611.06567.

[58] L. Lugiato, Introduction to the Feature Section on Cavity Solitons: An Overview, IEEE J. Quantum Electron. 39, 193 (2003).

[59] P. Parra-Rivas, D. Gomila, M. A. Matías, S. Coen, and L. Gelens, Dynamics of Localized and Patterned Structures in the Lugiato-Lefever Equation Determine the Stability and Shape of Optical Frequency Combs, Phys. Rev. A 89, 043813 (2014).

[60] S. Coen and M. Erkintalo, Universal Scaling Laws of Kerr Frequency Combs, Opt. Lett. 38, 1790 (2013). 
[61] T. Hansson and S. Wabnitz, Frequency Comb Generation Beyond the Lugiato-Lefever Equation: Multi-Stability and Super Cavity Solitons, J. Opt. Soc. Am. B 32, 1259 (2015).

[62] P. Del'Haye, T. Herr, E. Gavartin, M. L. Gorodetsky, R. Holzwarth, and T. J. Kippenberg, Octave Spanning Tunable Frequency Comb from a Microresonator, Phys. Rev. Lett. 107, 063901 (2011).

[63] M. Haelterman, S. Trillo, and S. Wabnitz, Dissipative Modulation Instability in a Nonlinear Dispersive Ring Cavity, Opt. Commun. 91, 401 (1992).

[64] J. K. Jang, M. Erkintalo, K. Luo, G.-L. Oppo, S. Coen, and S. G. Murdoch, Controlled Merging and Annihilation of Localised Dissipative Structures in an AC-Driven Damped Nonlinear Schrödinger System, New J. Phys. 18, 033034 (2016).

[65] F. Copie, M. Conforti, A. Kudlinski, A. Mussot, and S. Trillo, Competing Turing and Faraday Instabilities in Longitudinally Modulated Passive Resonators, Phys. Rev. Lett. 116, 143901 (2016).

[66] K. Ikeda, Multiple-Valued Stationary State and Its Instability of the Transmitted Light by a Ring Cavity System, Opt. Commun. 30, 257 (1979).

[67] S. Coen and M. Haelterman, Modulational Instability Induced by Cavity Boundary Conditions in a Normally Dispersive Optical Fiber, Phys. Rev. Lett. 79, 4139 (1997).

[68] J. M. Dudley, G. Genty, and S. Coen, Supercontinuum Generation in Photonic Crystal Fiber, Rev. Mod. Phys. 78, 1135 (2006).

[69] R. H. Stolen, J. P. Gordon, W. J. Tomlinson, and H. A. Haus, Raman Response Function of Silica-Core Fibers, J. Opt. Soc. Am. B 6, 1159 (1989).

[70] R. Salem, M. A. Foster, and A. L. Gaeta, Application of Spacetime Duality to Ultrahigh-Speed Optical Signal Processing, Adv. Opt. Photonics 5, 274 (2013).

[71] Each simulation is iterated for 10000 round trips (corresponding to more than 3000 photon lifetimes) to ensure steady state. The structures shown in Figs. 1(b) and 1(c) repeat identically from round trip to round trip, while asymmetric states with coexisting nonlinear structures [Figs. 1(d) and 1(e)] exhibit a trivial temporal drift in the simulation reference frame.

[72] X. Hachair, F. Pedaci, E. Caboche, S. Barland, M. Giudici, J. R. Tredicce, F. Prati, G. Tissoni, R. Kheradmand, L. A. Lugiato, I. Protsenko, and M. Brambilla, Cavity Solitons in a Driven VCSEL Above Threshold, IEEE J. Sel. Top. Quantum Electron. 12, 339 (2006).

[73] T. Hansson and S. Wabnitz, Bichromatically Pumped Microresonator Frequency Combs, Phys. Rev. A 90, 013811 (2014).

[74] M. Pesch, E. Große Westhoff, T. Ackemann, and W. Lange, Observation of a Discrete Family of Dissipative Solitons in a Nonlinear Optical System, Phys. Rev. Lett. 95, 143906 (2005).

[75] R. Neubecker, G.-L. Oppo, B. Thuering, and T. Tschudi, Pattern Formation in a Liquid-Crystal Light Valve with Feedback, Including Polarization, Saturation, and Internal Threshold Effects, Phys. Rev. A 52, 791 (1995).
[76] Y. Xu and S. Coen, Experimental Observation of the Spontaneous Breaking of the Time-Reversal Symmetry in a Synchronously Pumped Passive Kerr Resonator, Opt. Lett. 39, 3492 (2014).

[77] S. M. J. Kelly, Characteristic Sideband Instability of Periodically Amplified Average Soliton, Electron. Lett. 28, 806 (1992).

[78] Y. Wang, F. Leo, J. Fatome, M. Erkintalo, S. G. Murdoch, and S. Coen, Universal Mechanism for the Binding of Temporal Cavity Solitons, Optica 4, 855 (2017).

[79] See Supplemental Material at http://link.aps.org/ supplemental/10.1103/PhysRevX.7.031031 for corroborating evidence on the origins of fine features on CS spectra, as well as for statistical details related to the excitation and lifetimes of coexisting CS states.

[80] See Supplemental Material at http://link.aps.org/ supplemental/10.1103/PhysRevX.7.031031 for a full animation of the simulation results in Figs. 3(b) and 3(c).

[81] M. H. Anderson, F. Leo, M. J. Erkintalo, S. Coen, and S. G. Murdoch, in Photonics and Fiber Technology 2016 (ACOFT, BGPP, NP), OSA Technical Digest (online) (Optical Society of America, 2016), paper NW4A.4, http://www .osapublishing.org/abstract.cfm?uri=NP-2016-NW4A.4

[82] J. K. Jang, M. Erkintalo, S. G. Murdoch, and S. Coen, Writing and Erasing of Temporal Cavity Solitons by Direct Phase Modulation of the Cavity Driving Field, Opt. Lett. 40, 4755 (2015).

[83] See Supplemental Material at http://link.aps.org/ supplemental/10.1103/PhysRevX.7.031031 for a full animation of the simulation results in Fig. 4.

[84] Although the detuning is not locked, it can be assumed constant over the 0.5 -ms measurement time.

[85] F. Leo, T. Hansson, I. Ricciardi, M. De Rosa, S. Coen, S. Wabnitz, and M. Erkintalo, Walk-Off-Induced Modulation Instability, Temporal Pattern Formation, and Frequency Comb Generation in Cavity-Enhanced Second-Harmonic Generation, Phys. Rev. Lett. 116, 033901 (2016).

[86] T. Hansson, F. Leo, M. Erkintalo, S. Coen, I. Ricciardi, M. De Rosa, and S. Wabnitz, Singly Resonant SecondHarmonic-Generation Frequency Combs, Phys. Rev. A 95, 013805 (2017).

[87] P. Del'Haye, A. Coillet, W. Loh, K. Beha, S. B. Papp, and S. A. Diddams, Phase Steps and Resonator Detuning Measurements in Microresonator Frequency Combs, Nat. Commun. 6, 5668 (2015).

[88] J. P. Gordon, Theory of the Soliton Self-Frequency Shift, Opt. Lett. 11, 662 (1986).

[89] X. Yi, Q.-F. Yang, K. Y. Yang, and K. Vahala, Theory and Measurement of the Soliton Self-Frequency Shift and Efficiency in Optical Microcavities, Opt. Lett. 41, 3419 (2016).

[90] Y. V. Kartashov, O. Alexander, and D. V. Skryabin, Multistability and Coexisting Soliton Combs in Ring Resonators: The Lugiato-Lefever Approach, Opt. Express 25, 11550 (2017).

[91] M. Conforti and F. Biancalana, The Multi-Resonant Lugiato-Lefever Model, arXiv:1705.11086. 